\title{
Predictive values of genomic variation, tumor mutational burden, and PD-L1 expression in advanced lung squamous cell carcinoma treated with immunotherapy
}

\author{
Yanjun $\mathrm{Xu}^{1}$, Hui Li ${ }^{1}$, Zhiyu Huang ${ }^{1}$, Kaiyan Chen ${ }^{1}$, Xiaoqing Yu ${ }^{1}$, Jiamin Sheng ${ }^{1}$, Han-Han Zhang ${ }^{2}$, \\ Yun Fan $^{3}$ \\ ${ }^{1}$ Department of Medical Thoracic Oncology, Cancer Hospital of the University of Chinese Academy of Sciences, Zhejiang Cancer Hospital, Institute \\ of Cancer Research and Basic Medical Sciences of Chinese Academy of Sciences, Hangzhou, China; ${ }^{2}$ Burning Rock Biotech, Guangzhou, China; \\ ${ }^{3}$ Wenzhou Medical University, Wenzhou, China \\ Contributions: (I) Conception and design: Y Xu, Y Fan; (II) Administrative support: Z Huang, Y Fan; (III) Provision of study materials or patients: \\ Y Xu, Z Huang, K Chen; (IV) Collection and assembly of data: Y Xu, H Li, X Yu, J Sheng; (V) Data analysis and interpretation: Y Xu, H Li, HH \\ Zhang; (VI) Manuscript writing: All authors; (VII) Final approval of manuscript: All authors. \\ Correspondence to: Yun Fan. Wenzhou Medical University, Cancer Hospital of University of Chinese Academy of Sciences, Zhejiang Cancer Hospital, \\ Institute of Cancer Research and Basic Medical Sciences of Chinese Academy of Sciences. No. 1 East Banshan Road, Gongshu District, Hangzhou \\ 310022, China. Email: fanyun@zjcc.org.cn.
}

Background: Immune checkpoint inhibitors (ICIs) prolong overall survival (OS) in patients with advanced lung squamous cell carcinoma (LUSC). However, predictive and prognostic factors related to ICIs in LUSC remain elusive. This study aimed to identify predictors that are related to better clinical benefit and outcomes in LUSC patients treated with immunotherapy.

Methods: Capture-based targeted sequencing was performed in 64 patients with advanced LUSC who underwent immunotherapy. Tumor mutational burden (TMB) was defined as the sum of nonsynonymous single nucleotide and indel variants. Programmed cell death ligand-1 (PD-L1) expression was evaluated by immunohistochemical analysis. Clinicopathological characteristics including age, sex, performance status, smoking history, body mass index (BMI), blood fat, brain metastases, liver metastases, previous thoracic radiotherapy, and treatment lines were analyzed.

Results: The most commonly mutated genes included TP53, CDKN2A, KEAP1, CREBBP, KRAS, BIM, $A M E R 1$, and $A P C$. Copy number variations most frequently occurred in $A R, S O X 2, P I K 3 C A, E G F R$, RICTOR, FGFR1, and ZNF703. The median and mean TMB was 9.35 and 10.62 mutations per megabase, respectively. Positive PD-L1 expression was detected in $29.7 \%$ patients. Patients with a history of heavy smoking ( $\geq 40$ pack-years) were more likely to have positive $\mathrm{PD}-\mathrm{L} 1$ expression $(35 \%$ s. $16.7 \%, \mathrm{P}=0.04)$ and higher TMB (11.1 vs. 9.8 mut/Mb, $\mathrm{P}=0.04)$. Gene alterations had no impact on PD-L1 expression or TMB level. The median progression-free survival (PFS) was 6.7 months and median OS was 13.7 months. Higher TMB was independently associated with longer PFS $(\mathrm{P}=0.01)$ and $\mathrm{OS}(\mathrm{P}=0.02)$, and this correlation was more pronounced in patients treated with ICIs as a single agent $(\mathrm{P}=0.0001)$. Higher TMB was also associated with better disease control rate (DCR) $(\mathrm{P}=0.02)$. Compared with wild-type, patients with KRAS mutation and $E G F R$ amplification had higher objective response rates (ORR, $\mathrm{P}=0.01$ ).

Conclusions: The predictive value of TMB is more significant in LUSC patients receiving ICI as a single agent than as a combination therapy. The combination of Eastern Cooperative Oncology Group performance status (ECOG-PS), smoking status, TMB, PD-L1, and genomic variation might be helpful for personalized immunotherapy decisions in clinical practice for advanced LUSC.

Keywords: Genome; immunotherapy; lung squamous cell carcinoma (LUSC); programmed cell death ligand-1 (PD-L1); tumor mutational burden (TMB) 
Submitted Sep 15, 2020. Accepted for publication Nov 17, 2020.

doi: $10.21037 /$ tlcr-20-1130

View this article at: http://dx.doi.org/10.21037/tlcr-20-1130

\section{Introduction}

Lung squamous cell carcinoma (LUSC) accounts for $30 \%$ of all lung cancers (1). Although the therapeutic management for metastatic non-squamous-cell carcinoma has revolutionized from chemotherapy to moleculartargeted therapy in the past decade, platinum-based chemotherapy remains the first-line standard treatment for advanced LUSC (2), due to a lack of therapeutic targets. LUSC offers a very different genetic picture.

Recently, significant progress has been made in the development of immune checkpoint inhibitors (ICIs) $(3,4)$, which target the programmed cell death-1 (PD-1)programmed cell death ligand-1 (PD-L1) axis and elicit remarkable clinical efficacy in both treatment naïve and previously-treated advanced or metastatic non-small cell lung cancer (NSCLC) patients. According to the National Comprehensive Cancer Network (NCCN) guidelines, the recommended first-line treatment for advanced LUSC with PD-L1 expression levels of $\geq 50 \%$ is pembrolizumab monotherapy, and for patients with metastatic LUSC is pembrolizumab/carboplatin/paclitaxel (or albumin-bound paclitaxel) (4-6). Multiple trials have demonstrated a superior progression-free survival (PFS) and overall survival (OS) in advanced or metastatic LUSC, who received pembrolizumab either as a single agent or in combination with standard chemotherapy (4). Moreover, several ICIs including pembrolizumab, nivolumab, and atezolizumab, have also been approved as second-line treatment regimens for patients with advanced or metastatic LUSC (7-9).

However, responses to ICIs therapies occur in only a minority of NSCLC patients. Identification of predictive biomarkers of response seems emergent. Biomarker studies have revealed some genomic features that are predictive of the efficacy of immunotherapy including (but not limited to) microsatellite instability (MSI) status, positive PDL1 expression, and high tumor mutational burden (TMB) $(10,11)$. However, unlike adenocarcinoma patients, a considerable proportion of LUSC patients with advanced stage refused to provide tumor tissue for molecular testing. Moreover, challenges in defining appropriate cut-off values and standardizing the testing platform have limited their clinical application.
Therefore, the ability of genomic features, TMB, PDL1 expression, or other clinical characteristics to be used as predictive factors to guide immunotherapies in realworld clinical practice has attracted tremendous attention. Recent reports have indicated that gene profiling, cytokines, Eastern Cooperative Oncology Group performance status (ECOG-PS), and body mass index (BMI) were significant in predicting the response to immunotherapy $(12,13)$, however each of these offers limited utility. A similar study has been carried out in NSCLC including adenocarcinoma and squamous cell carcinoma treated with combined anti-CTLA-4 and anti-PD-1 therapies (14). But the predictive value of mentioned indicators in the immunotherapy of LUSC is unclear. Studies have suggested that there are much more differences in the response of LUSC and adenocarcinoma to immunotherapy, as well as gene status and immune microenvironment. LUSC is not well characterized due to the poor understanding of the genomic evolution (15). Genomic alterations in the tumor characterize also various in different stages of LUSC progression. Thus, in particular, we focused on advanced LUSC. In this study, tissue samples and complete survival follow-up of patients with advanced LUSC who received immunotherapy in real-world clinical practice were examined to identify ideal factors related to better efficacy and prognosis. We present the following article in accordance with the REMARK reporting checklist (available at http://dx.doi.org/10.21037/tlcr-20-1130).

\section{Methods}

\section{Patients and samples}

A total of 911 patients with NSCLC who received antiPD-L1 therapeutic agents at the Cancer Hospital of the University of Chinese Academy of Sciences (Zhejiang Cancer Hospital) between January 2016 and June 2019 were screened retrospectively. Only advanced and metastatic LUSC patients with sufficient archived tissue samples and complete survival data were included. The major inclusion criteria were as follows: >18 years old; an ECOG-PS of $0-2$; have advanced or recurrent LUSC; treated with antiPD-L1 therapy agents; radiologically evaluable according 


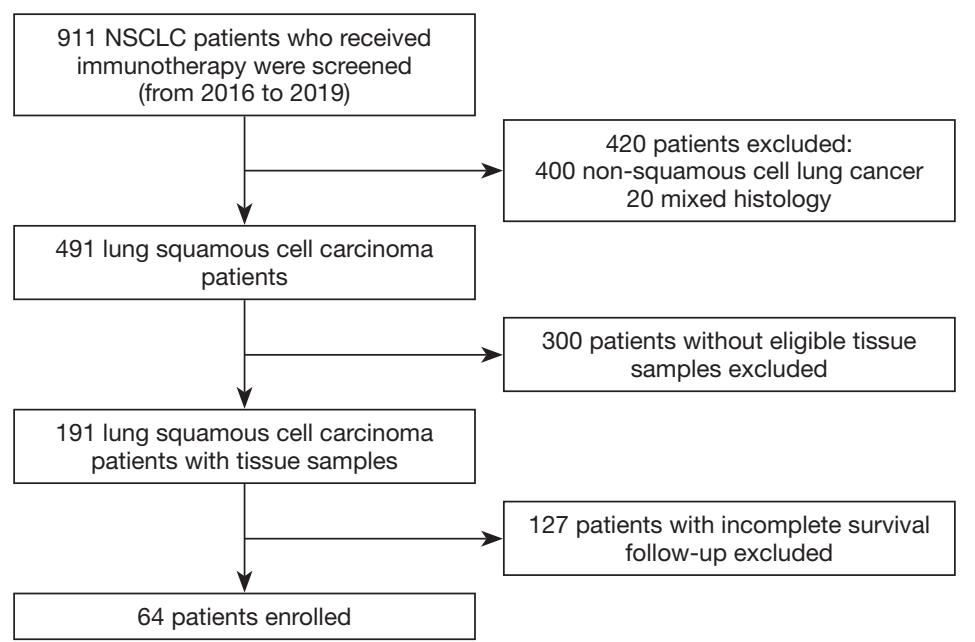

Figure 1 Trial profile. A total of 911 patients with NSCLC who underwent immunotherapy were screened. Major exclusion criteria were inadequate tissue samples, mixed histology, and incomplete follow-up data. Sixty-four patients were finally enrolled. NSCLC, non-small cell lung cancer.

to Response Evaluation Criteria in Solid Tumors (RECIST) version 1.1; a minimum of $50 \%$ of tumor content was required. The major exclusion criteria were as follows: inadequate tissue samples; non-squamous histology; incomplete follow-up data. A total of 64 patients who met the inclusion criteria were enrolled.

Formalin-fixed and paraffin-embedded tissues were used for capture-based targeted sequencing and immunohistochemical (IHC) staining. The study scheme is summarized in Figure 1. The following clinicopathological features were analyzed: age, sex, ECOG-PS, smoking history, BMI, blood fat, brain metastases, liver metastases, history of thoracic radiotherapy, and treatment lines. PFS was defined as the time from the initiation of immunotherapy until disease progression. OS was calculated from the date of the initiation of immunotherapy until death of any cause or the last follow-up date. All procedures performed in studies involving human participants were in accordance with the Helsinki Declaration (as revised in 2013). The study was approved by the ethics committee of Zhejiang Cancer Hospital. Informed consent was obtained from each patient.

\section{Capture-based targeted sequencing}

DNA shearing was performed on tissue DNA using Covaris M220, followed by end repair, phosphorylation and adaptor ligation. Fragments of size 200-400 bp were selected by bead (Agencourt AMPure XP Kit), followed by hybridization with capture probes baits, hybrid selection with magnetic beads and PCR amplification. Indexed samples were sequenced on Nextseq500 sequencer (Illumina, Inc., USA) with pair-end reads. Capture-based targeted sequencing for somatic mutation profiling was performed using a panel consisting of 520 cancer related genes, spanning $1.7 \mathrm{Mb}$ of human genome.

\section{TMB calculation}

TMB was defined as the number of somatic single nucleotide variants (SNVs), coding, base substitution, and indels per megabase of genome examined, and was calculated as previously described (16). Briefly, all base substitutions, including non-synonymous and synonymous alterations, and indels in the coding region of targeted genes were considered with the exception of known hotspot mutations in oncogenic driver genes and truncations in tumor suppressors. Synonymous mutations were counted in order to reduce sampling noise.

\section{PD-L1 expression}

PD-L1 expression was tested by anti-human PD-L1 (Dako $22 \mathrm{C} 3$ ) according to the manufacturer's recommendations, using 4-5 $\mu \mathrm{m}$ formalin-fixed and paraffin-embedded (FFPE) sections. The cutoff value was $1 \%$ for PD-L1 positivity or 
negativity (PD-L1+/-). Representative IHC images have been provide as supplemented Figure S1.

\section{Statistical analysis}

Spearman's rank correlation was used to assess the correlations among gene variation, PD-L1 expression, $\mathrm{TMB}$, and clinical parameters. Continuous variables were analyzed using analysis of variance and Tukey's multiple comparison tests. Discrete variables were analyzed using the Chi-square test or Fisher's exact test. The KaplanMeier curve was leveraged to assess the patients' survival curves. The Cox proportional-hazards model was used for univariate and multivariate survival analyses to calculate the hazard ratios (HR) and related $95 \%$ confidence intervals (CIs) for PFS and OS times. Logistic regression was used for univariate and multivariate analyses to calculate the odds ratios and related $95 \%$ CIs for overall response and disease control. A P value less than 0.05 was considered significant. All statistical analyses were performed using the SAS software, version 9.4 (SAS Institute Inc., NC, USA).

\section{Results}

Clinicopathological characteristics of advanced LUSC and their correlations with TMB and PD-L1 expression

A total of 64 patients with advanced and metastatic LUSC who were treated with anti-PD-L1 therapy agents at Zhejiang Cancer Hospital between January 2016 and November 2019 were included. All patients were stage IV at diagnosis with a median age of 62 years (range, $45-81$ years) at the time of treatment initiation, and $92.2 \%$ were men. Also, $96.9 \%$ of patients had an ECOG-PS of between 0 and 1 .

Thirteen $(20.3 \%)$ patients received immunotherapy as first-line, $29(45.3 \%)$ as second-line, and the remaining $34.4 \%$ of patients as > second-line treatment. Eight (12.5\%) patients had brain metastasis, 14 (21.9\%) had liver metastasis, and 35 (54.7\%) had dyslipidemia. Thirty-five $(54.7 \%)$ patients had a history of thoracic radiotherapy. Fifty-three $(82.8 \%)$ patients received monoimmunotherapy. The median BMI was 22.24. The median and mean TMB was 9.35 and $10.62 \mathrm{mut} / \mathrm{Mb}$, respectively. A PD-L1 tumor proportion score (TPS) $>1 \%$ was considered as PD-L1 positivity and was identified in $29.7 \%$ of patients with LUSC (Table 1). No statistically significant difference was found in the clinicopathological characteristics, including age, sex, brain metastases, liver metastases, history of thoracic radiotherapy, and treatment lines between patients with high $(>10.62 \mathrm{mut} / \mathrm{Mb})$ or low $(\leq 10.62 \mathrm{mut} / \mathrm{Mb})$ TMB or PD-L1 positivity.

Patients with a history of heavy smoking ( $\geq 40$ packyears) were more likely to have positive PD-L1 expression ( $35 \%$ vs. $16.7 \%, \mathrm{P}=0.04$ ) and higher TMB (11.1 vs. $9.8 \mathrm{mut} / \mathrm{Mb}, \mathrm{P}=0.04)$. Patients with a $\mathrm{BMI}$ of $18.5-24$ exhibited a trend of having a higher percentage of positive PD-L1 expression $(\mathrm{P}=0.02)$ and a lower TMB level $(\mathrm{P}=0.04)$. The baseline characteristics of patients in this cohort and their correlations with TMB and PD-L1 expression are summarized in Table 1. The proportion of LUSC patients with positive PD-L1 and high TMB increases with the addition of smoking pack-years (Table 1 and Figure S2). Positive PD-L1 expression was identified in $14.29 \%$ of never smokers, $23.53 \%$ of $<40$ pack-year smokers, and $35 \%$ of $\geq 40$ pack-year smokers. High TMB was identified in $28.57 \%$ of never smokers, $41.18 \%$ of $<40$ pack-year smokers, and $50 \%$ of $\geq 40$ pack-year smokers (Figure S2).

\section{Gene variations of advanced LUSC and their correlations with TMB and PD-L1 expression}

Next, we investigated the correlations between frequently mutated genes and TMB/PD-L1 status. Eight genes with a somatic mutation frequency $>5 \%$ were identified: TP53, CDKN2A, KEAP1, CREBBP, KRAS, BIM, AMER1, and $A P C$ (Figure 2). Genes with copy number variations occurring in $>5 \%$ patients were also investigated, and seven genes were identified: $A R, S O X 2, P I K 3 C A, E G F R$, RICTOR, FGFR1, and ZNF703 (Figure 2). Mutation in the aforementioned genes had no impact on PD-L1 expression or TMB level (Figure 2, Table S1).

\section{Predictive and prognostic value of clinicopatbological characteristics}

The median follow-up time was 17 months. The disease control rate (DCR) was 81.3\% [partial response (PR) 29.7\% + stable disease (SD) $51.6 \%$ ]. The objective response rate (ORR) was $29.2 \%$, with a median PFS of 6.7 months and a median OS of 13.7 months. The 6-month PFS rate was $57.8 \%$, and the 1 -year OS rate was $62.5 \%$ (Figure 3).

Better ECOG-PS was associated with longer PFS $(\mathrm{P}=0.019)$ (Table 2, Figure 4), and also with a better ORR $(\mathrm{P}<0.0001)$ (Table S2). Our data revealed that patients with an ECOG-PS of 0 were more likely not to have disease progression at 6-month $(\mathrm{P}<0.005)$. The 6-month $\mathrm{PFS}$ rate 
Table 1 Baseline characteristics of included patients and their correlations with TMB and PD-L1 expression (N=64)

\begin{tabular}{|c|c|c|c|c|c|c|c|c|c|c|c|}
\hline Characteristic & $\begin{array}{l}\text { Total } \\
\text { N (\%) }\end{array}$ & \multicolumn{2}{|c|}{ PD-L1 (-) } & \multicolumn{2}{|c|}{ PD-L1 (+) } & $\mathrm{P}$ value & \multicolumn{2}{|c|}{ TMB low* } & \multicolumn{2}{|c|}{ TMB high $^{\star *}$} & $P$ value \\
\hline \multicolumn{12}{|l|}{ Age } \\
\hline$<65$ & $41(64.1)$ & 28 & 68.29 & 13 & 31.71 & 0.6368 & 23 & 56.1 & 18 & 43.90 & 0.7622 \\
\hline$\geq 65$ & $23(35.9)$ & 17 & 73.91 & 6 & 26.09 & & 12 & 52.17 & 11 & 47.83 & \\
\hline Male & $59(92.2)$ & 41 & 69.49 & 18 & 30.51 & 0.3713 & 31 & 52.54 & 28 & 47.46 & 0.1992 \\
\hline Female & $5(7.8)$ & 4 & 80 & 1 & 20 & & 4 & 80 & 1 & 20 & \\
\hline \multicolumn{12}{|l|}{$\mathrm{BMI}$} \\
\hline$<18.5$ & $7(10.9)$ & 5 & 71.43 & 2 & 28.57 & 0.0168 & 3 & 42.86 & 4 & 57.14 & 0.0436 \\
\hline \multicolumn{12}{|l|}{ Smoking } \\
\hline Never & 7 (10.9) & 6 & 85.71 & 1 & 14.29 & 0.0443 & 5 & 71.43 & 2 & 28.57 & 0.0405 \\
\hline \multicolumn{12}{|l|}{ Current/former } \\
\hline$<40$ pack-years & 17 (26.6) & 13 & 76.47 & 4 & 23.53 & & 10 & 58.82 & 7 & 41.18 & \\
\hline$\geq 40$ pack-years & $40(62.5)$ & 26 & 65 & 14 & 35 & & 20 & 50 & 20 & 50 & \\
\hline \multicolumn{12}{|l|}{ ECOG } \\
\hline 0 & $18(28.1)$ & 12 & 66.67 & 6 & 33.33 & 0.1105 & 9 & 50 & 9 & 50 & 0.2325 \\
\hline 1 & 44 (68.8) & 31 & 70.45 & 13 & 29.55 & & 26 & 59.09 & 18 & 40.91 & \\
\hline \multicolumn{12}{|l|}{ Brain metastases } \\
\hline Yes & $8(12.5)$ & 6 & 75 & 2 & 25 & 0.3147 & 5 & 62.5 & 3 & 37.5 & 0.268 \\
\hline No & 56 (87.5) & 39 & 69.64 & 17 & 30.36 & & 30 & 53.57 & 26 & 46.43 & \\
\hline \multicolumn{12}{|l|}{ Liver metastases } \\
\hline Yes & $14(21.9)$ & 11 & 78.57 & 3 & 21.43 & 0.2055 & 7 & 50 & 7 & 50 & 0.6902 \\
\hline No & $50(78.1)$ & 34 & 68 & 16 & 32 & & 28 & 56 & 22 & 44 & \\
\hline \multicolumn{12}{|l|}{ Radiotherapy } \\
\hline Yes & $35(54.7)$ & 26 & 74.29 & 9 & 25.71 & 0.4447 & 17 & 48.57 & 18 & 51.43 & 0.2802 \\
\hline No & $29(45.3)$ & 19 & 65.52 & 10 & 34.48 & & 18 & 62.07 & 11 & 37.93 & \\
\hline \multicolumn{12}{|l|}{ Lines of therapy } \\
\hline$\leq 2$ & $42(65.6)$ & 29 & 69.05 & 13 & 30.95 & 0.7596 & 23 & 54.76 & 19 & 45.24 & 0.9868 \\
\hline$>2$ & $22(34.4)$ & 16 & 72.73 & 6 & 27.27 & & 12 & 54.55 & 10 & 45.45 & \\
\hline
\end{tabular}

*, TMB low, TMB $\leq 10.62$ (Mean TMB for all 64 subjects is 10.62); ${ }^{\star *}$, TMB high, TMB >10.62. ECOG PS, Eastern Cooperative Oncology Group performance score; PD-L1, programmed cell death ligand-1; TMB, tumor mutational burden. 


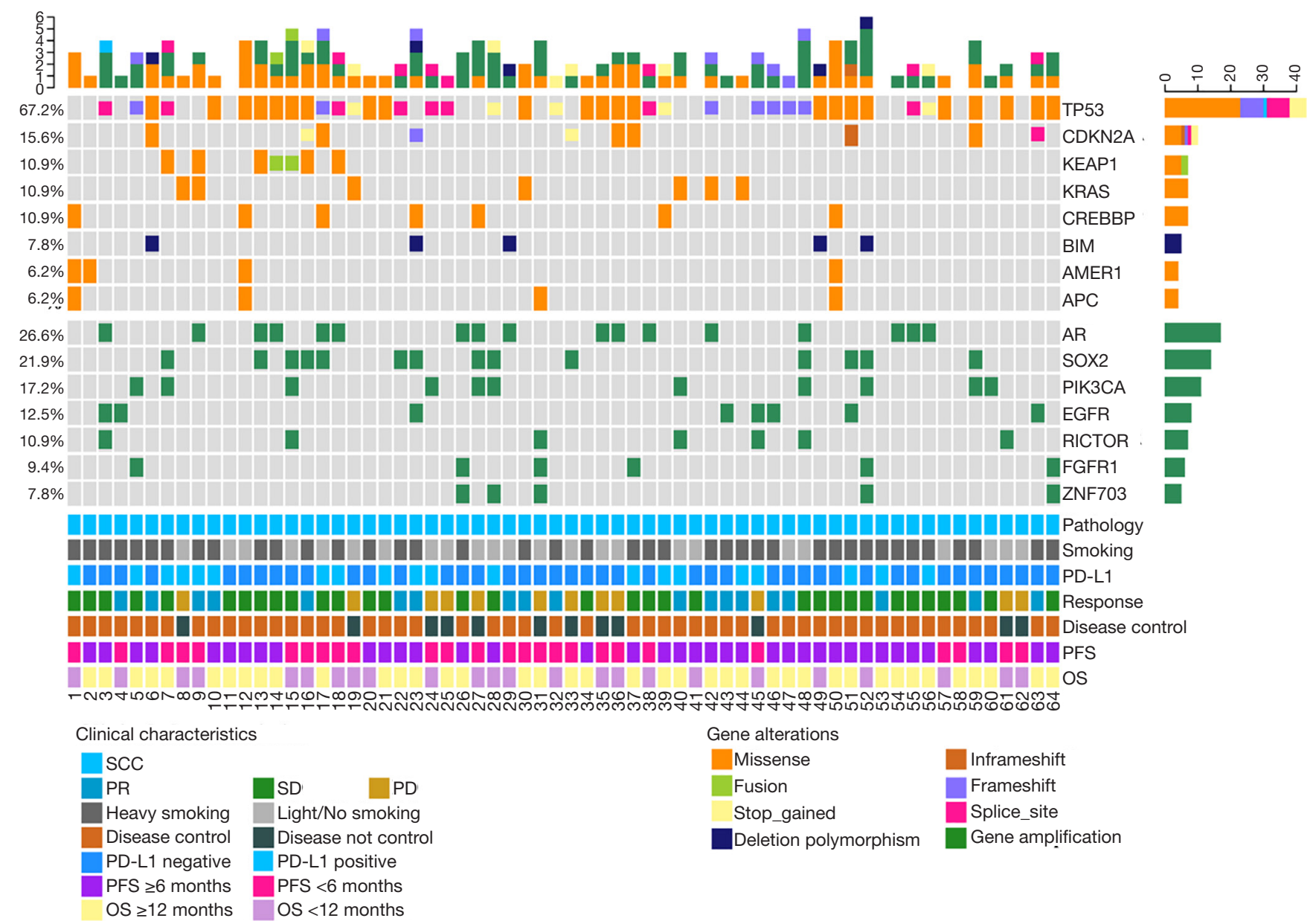

Figure 2 Gene variations of advanced LUSC and their correlations with clinical characteristics. Eight genes with a somatic mutation frequency $>5 \%$ were identified: TP53, CDKN2A, KEAP1, CREBBP, KRAS, BIM, AMER1, and APC. Seven genes with copy number variation frequency $>5 \%$ were as follows: AR, SOX2, PIK3CA, EGFR, RICTOR, FGFR1, and ZNF703. Each column represents one patient. Clinical characteristics and genetic alterations of each patient were shown at the bottom. LUSC, lung squamous cell carcinoma; PR, partial response; $\mathrm{SD}$, stable disease; $\mathrm{PD}$, progressive disease.
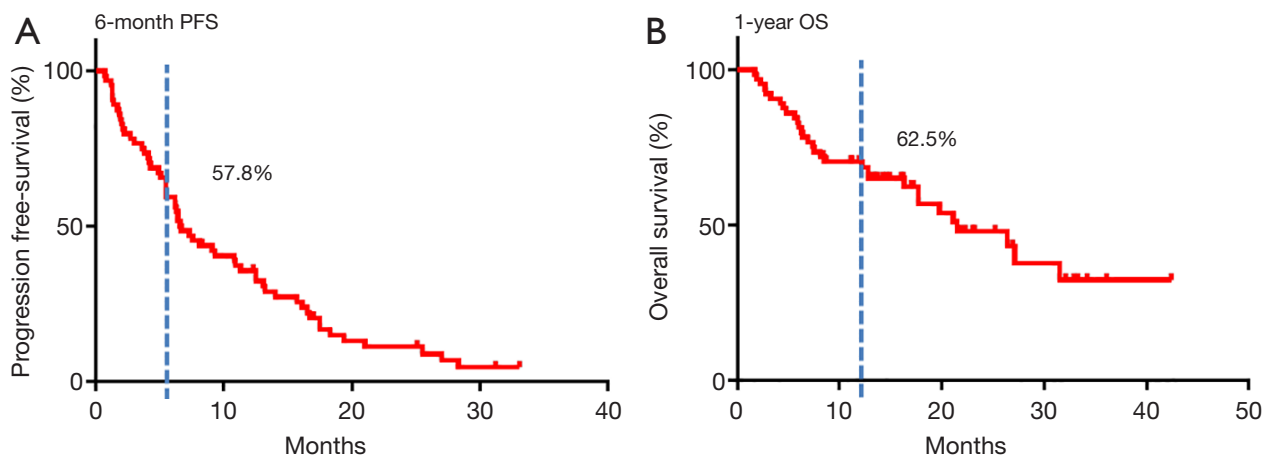

Figure 3 Kaplan-Meier estimates for progression-free survival and overall survival (N=64). (A) The median PFS was 6.7 months. The 6-month PFS rate was $57.8 \%$. (B) The median OS was 13.7 months. The 1 -year OS rate was $62.5 \%$. PFS, progression-free survival; OS, overall survival. 
Table 2 Univariate and multivariate analyses of clinical parameters on progression-free survival (PFS) and overall survival (OS) (Cox regression) $(\mathrm{N}=64)$

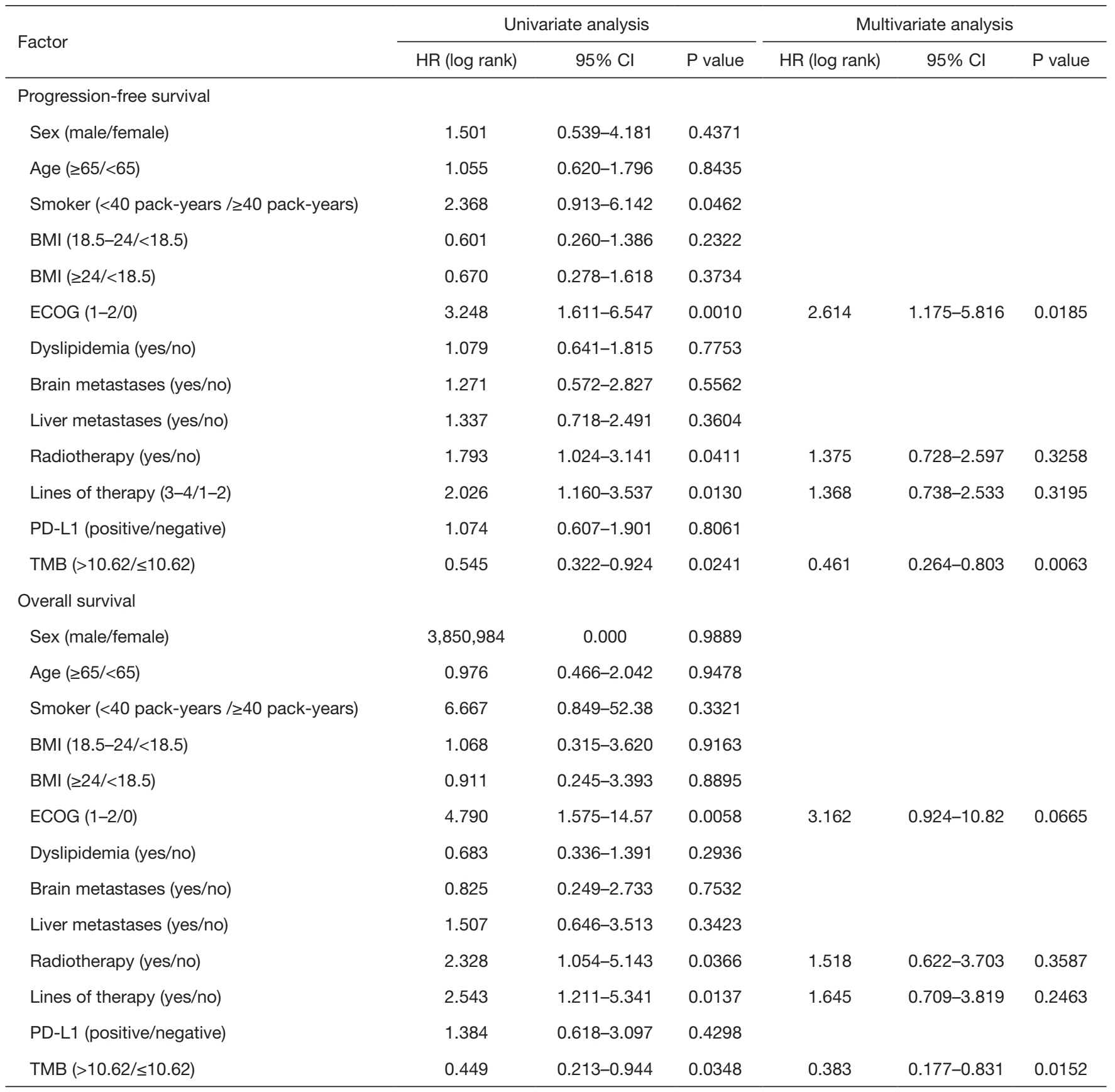

BMI, body mass index; ECOG, Eastern Cooperative Oncology Group; PD-L1, programmed cell death ligand-1; TMB, tumor mutational burden.

in patients with an ECOG-PS of 0 and $1-2$ was $72.2 \%$ and $54.3 \%$, respectively (Figure $4 A$ ). The 1-year OS rate showed a higher trend in patients with an ECOG-PS of 0 compared to patients with an ECOG-PS of $1-2$ (80.5\% vs. $65.2 \%$,
$\mathrm{P}=0.067$ ) (Figure 4C).

Considering the impact of smoking history on TMB and a high percentage of heavy smokers $(62.5 \%, \mathrm{~N}=40)$ in this cohort, an analysis of smoking history and clinical outcomes 

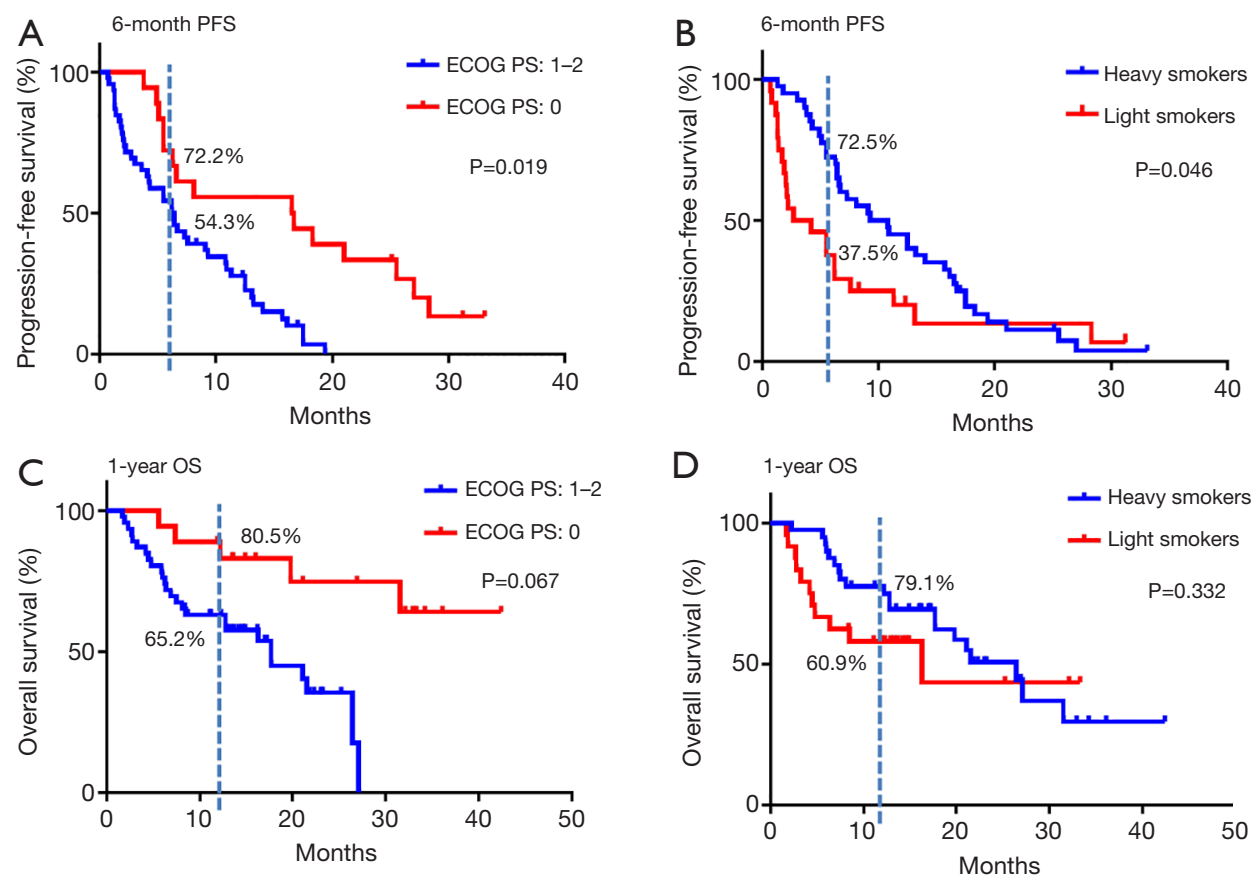

Figure 4 Prognostic value and effectiveness of clinicopathological characteristics. (A) The 6-month PFS rate in patients with an ECOG PS of 0 and $1-2$ was $72.2 \%$ and $54.3 \%$, respectively $(\mathrm{P}=0.019)$. (B) The 6-month PFS rate was $72.5 \%$ and $37.5 \%$ in heavy smokers $(\geq 40$ packyears) and light smokers, respectively $(\mathrm{P}=0.046)$. (C) The 1-year OS rate in patients with an ECOG PS of 0 and $1-2$ was $80.5 \%$ and $65.2 \%$, respectively $(\mathrm{P}=0.067)$. (D) No statistically significant difference was found in the relationship of smoking status with OS (P=0.332). The 1-year OS rate was $79.1 \%$ and $60.9 \%$ in heavy smokers ( $\geq 40$ pack-years) and light smokers, respectively. ECOG PS, Eastern Cooperative Oncology Group performance score; PFS, progression-free survival; OS, overall survival.

was conducted. Patients with a history of heavy smoking ( $\geq 40$ pack-years) were more likely to have a longer PFS $(\mathrm{P}<0.005)$, but were not statistically different in OS (Figure $4 B, D)$. The 6-month PFS rates for heavy smokers and non-heavy smokers were $72.5 \%$ and $37.5 \%$, respectively $(\mathrm{P}=0.046)$. The 1 -year OS rates for heavy smokers and non-heavy smokers were $79.1 \%$ and $60.9 \%$, respectively $(\mathrm{P}=0.332)$. However, patients with a history of heavy smoking had a better DCR (97.5\% vs. 54.2\%, $\mathrm{P}=0.01$ ) (Table S2).

Moreover, the univariate analysis revealed that a history of thoracic radiotherapy was associated with a better ORR ( $\mathrm{P}=0.001)$ (Table S2). However, according to the multivariate analysis, the difference was not statistically significant. Similar results were observed between lines of therapy and DCR (Table S2). In the total population, there were $53(82.8 \%)$ patients who received immunotherapy as a single agent (mono-immunotherapy group, $\mathrm{N}=53$ ). According to the multivariate analysis of this monoimmunotherapy group, BMI (18.5-24 vs. $<18.5, \mathrm{P}=0.023)$ and lines of therapy $(3-4 v s .1-2, \mathrm{P}=0.005)$ were found to be associated with PFS (Table S3).

\section{Predictive and prognostic value of TMB and PD-L1 expression}

The median and mean TMB in this cohort was 9.35 and $10.62 \mathrm{mut} / \mathrm{Mb}$, respectively. Higher TMB was defined as $>10.62 \mathrm{mut} / \mathrm{Mb}$ (Table 1), which was found to be independently associated with longer PFS $(\mathrm{P}=0.006)$ and $\mathrm{OS}$ $(\mathrm{P}=0.015)$ (Table 2, Figure $5 A, B)$. This correlation was more pronounced in patients treated with mono-immunotherapy $(\mathrm{P}=0.0001)$ (Figure 5C,D, Table S3). Patients with a higher TMB level had a better DCR in both the total population and the mono-immunotherapy group $(\mathrm{P}=0.02)$ (Table S2). Univariate analyses of combined TMB and PD-L1 on OS and PFS showed that TMB has a greater predictive value for PFS in PD-L1 negative populations than PD-L1 positive populations, and were also more significant in the mono-immunotherapy group than the combined therapy group (Table S4). 

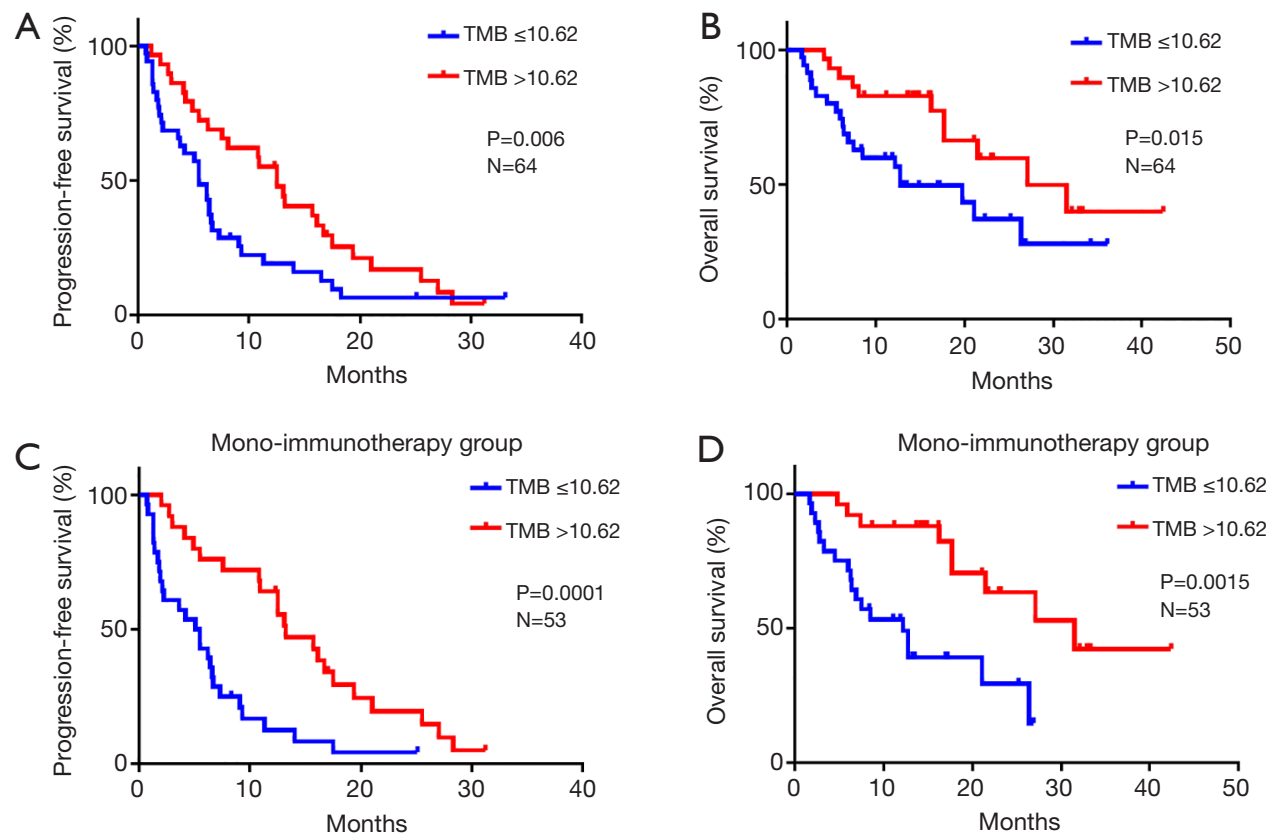

Figure 5 TMB correlate with PFS and OS in advanced LUSC receiving immunotherapy. (A) Improved PFS in all patients with high TMB (>10.62) $(\mathrm{N}=64, \mathrm{P}=0.006)$. (B) Improved OS in patients with high TMB (>10.62) $(\mathrm{N}=64, \mathrm{P}=0.015)$. A more significant trend toward increased PFS (N=53, $\mathrm{P}=0.0001)(\mathrm{C})$ and $\mathrm{OS}(\mathrm{N}=53, \mathrm{P}=0.0015)(\mathrm{D})$ was also observed in high TMB patients receiving mono-immunotherapy. TMB, tumor mutational burden; PFS, progression-free survival; OS, overall survival; LUSC, lung squamous cell carcinoma.

\section{Predictive and prognostic value of gene mutation and amplification}

The univariate and multivariate analyses revealed no statistically significant correlations between gene alterations and PFS or OS (Figure 2, Table S5). Moreover, KRAS mutation and $E G F R$ amplification were found to be associated with better ORR $(\mathrm{P}=0.01)$ (Figure 2, Figure S3, Table S6).

\section{Discussion}

This retrospective analysis explored potential predictive and prognostic biomarkers of ICIs in stage IV LUSC patients treated with immunotherapy as a single agent or a combination therapy. The median PFS and OS were 6.7 and 13.7 months in this cohort, respectively. The 6-month PFS rate was $57.8 \%$ and the 1 -year OS rate was $62.5 \%$. Thirty-three people survived until the last follow-up.

In the phase III study (CheckMate 017) of nivolumab $v s$. docetaxel in LUSC, the median PFS and OS for patients treated with nivolumab as the second-line were 3.5 and 9.2 months, respectively (7). After 1 year, the OS rate was $42 \%$ with nivolumab (7). In the double-blind phase 3 trial (Keynote-407) of pembrolizumab plus chemotherapy for LUSC, the median PFS was 6.4 months in the pembrolizumab combination group (17).

In contrast, $79.7 \%$ of the patients in the present study received immunotherapy as a second-line (or beyond) treatment; their median PFS, OS, and 1-year OS rates were comparable to those receiving nivolumab as a second-line treatment. It might not be appropriate to compare the data of a retrospective study directly with that of prospective clinical trials. However, these results could be explained by the fact that most of the patients included in the present analysis had an ECOG-PS score of 0-1 (96.9\%), and $40(62.5 \%)$ patients were heavy smokers. A real-world study in Canada indicated that patients with an ECOGPS score of $0-1$ benefited more significantly compared with those with an ECOG-PS score of 2, with median OS times of 12.91 and 6.77 months, respectively (18). Patients with an ECOG-PS of 2 might have been excluded from most immunotherapy trials. The available data suggested that patients with an ECOG-PS score of 2 had a worse prognosis, and that an ECOG-PS of 2 might be a predictor for shorter PFS and OS (18-22). 
When considering smoking history, previous studies have shown that tobacco was a major cause of genomic instability and heterogeneity in cancer, and smokers had a higher TMB (23). The EAP study in Italy showed a higher ORR in patients with long-term smoking history, and prolonged PFS was observed in patients with a smoking history of $>5$ pack-years compared with patients with a smoking history of $\leq 5$ pack-years (24). Moreover, objective responses were observed in $27 \%$ of never smokers, $40 \%$ of light smokers, and $40 \%$ of heavy smokers with NSCLC treated with immunotherapies (24). The $29.2 \%$ ORR in this study was closer to that achieved in the aforementioned studies using monotherapy in patients with LUSC. In the present study, patients with a history of heavy smoking tended to have a higher PD-L1 positive rate and higher TMB. The proportion of LUSC patients with positive PD-L1 and high TMB increases with the addition of smoking pack-years. Variables associated with a better ORR or DCR in the multivariate analysis included an ECOG-PS of 0 , a history of heavy smoking ( $\geq 40$ pack-years), and a high TMB. These results might partially explain the similarity between the present study and previous data.

In fact, treatment strategies for lung adenocarcinoma have significantly revolutionized, owing to the identification of targetable mutations (known as driver mutations) (25). However, less evidence is available regarding druggable genetic targets in LUSC. Attempts have been made using the next-generation sequencing (NGS) approach to identify driver mutations and identify those of clinical relevance to LUSC. Frequent mutations in TP53 (64.5\% of analyzed patients), PIK3CA (28.5\%), CDKN2A (24.4\%), SOX2 (17.7\%), and CCND1 (15.8\%) have been identified (26). The Cancer Genome Atlas (TCGA) has identified 10 genes (TP53, CDKN2A, PTEN, PIK3CA, KEAP1, MLL2, HLA-A, NFE2L2, NOTCH1, and RB1) with a Q value of $<0.1$ (27). In the present study, eight genes with a mutation rate of >5\% were identified: TP53, CDKN2A, KEAP1, CREBBP, $K R A S, B I M, A M E R 1$, and $A P C$. Seven genes had significant copy number variations: $A R, S O X 2, P I K 3 C A, E G F R$, RICTOR, FGFR1, and ZNF703. These results differed slightly from TCGA findings and the published data. However, no significant correlations were observed between gene variations and clinicopathological features, DCR, PFS, and OS in the multivariate analysis. Moreover, our study indicated that genomic alterations did not correlate with the TMB level.

Also, mutations associated with a better ORR in our study included the KRAS mutation and EGFR amplification.
Some recent studies suggested that patients with KRAS mutations might benefit from immunotherapy due to the association between smoking and KRAS mutations $(28,29)$. Other studies showed that co-occurring mutations in TP53 and KRAS might be associated with the activation of antitumor immunity $(30,31)$. Actually, the underlying mechanisms of TP53 and/or KRAS co-occurrence have not been explored yet. EGFR amplification is considered to be a negative prognostic factor in immunotherapy $(32,33)$. Contrary to previous studies, our results may be due to the small sample size and other confounding factors. The relationship between $E G F R$ amplification and the efficacy of immunotherapy remains to be explored.

In addition, this study explored the relationships between clinical features, genomic alterations, PD-L1 expression, and also TMB. The mean TMB was $10.62 \mathrm{mut} / \mathrm{Mb}$ and the median TMB was $9.35 \mathrm{mut} / \mathrm{Mb}$, which were slightly higher than TCGA findings (mean TMB, 8.1 mut/Mb; median TMB, $8.4 \mathrm{mut} / \mathrm{Mb}$ ) and similar to the CHOICE (mean TMB, $11.8 \mathrm{mut} / \mathrm{Mb}$ ) studies $(27,34)$. This may be explained due to a higher rate of heavy smokers in our cohort. LUSC was considered to have higher TMB because of tobacco exposure. A significant association was observed between smoking history and TMB in the present study with a large proportion of heavy smokers (34-37).

Currently, TMB and PD-L1 expression are identified to be the two major predictive biomarkers for anti-PD-1/PDL1 therapy. In our study, we found no association between TMB level and PD-L1 expression, which was consistent with previous data (38). Interestingly, our findings indicated that high TMB (>10.62 mut/Mb) was correlated with significantly longer PFS and OS in advanced LUSC and especially in patients who received mono-immunotherapy, which was also consistent with previous reports (11,39-41). TMB > $10.62 \mathrm{mut} / \mathrm{Mb}$ identified LUSC patients who may potentially benefit from mono-immunotherapy irrespective of PD-L1 expression and genomic alterations. Patients with a higher TMB level had a better DCR in both the total population and the mono-immunotherapy group. Moreover, univariate analyses of TMB and PD-L1 on OS and PFS showed that TMB has greater predictive value for PFS in PD-L1 negative populations and the monoimmunotherapy group.

\section{Conclusions}

TMB is predictive factor of improved clinical outcomes in advanced LUSC patients receiving immunotherapy, 
and the predictive value is more pronounced in patients treated with immunotherapy as a single agent. TMB seems to have greater predictive value for PFS in PD-L1 negative populations than in $\mathrm{PD}-\mathrm{L} 1$ positive populations. Although the small sample size and cohort heterogeneity might limit the conclusions made in this study, the combination of ECOG-PS, smoking status, TMB, PD-L1, and genomic alterations might be helpful for personalized immunotherapy decisions in clinical practice for advanced LUSC.

\section{Acknowledgments}

The authors appreciate technical support from $\mathrm{Mr}$. Yonggang Ding from Jiangsu Hengrui Medicine Co.

Funding: This study was supported by the Natural Scientific Foundation of China (No. 81972718), the Natural Scientific Foundation of Zhejiang Province, China (No. LY19H160007 and LGF18H160017), and the Science and Technology Program for Health and Medicine in Zhejiang Province, China (2021419564).

\section{Footnote}

Reporting Checklist: The authors have completed the REMARK reporting checklist. Available at http://dx.doi. org/10.21037/tlcr-20-1130

Data Sharing Statement: Available at http://dx.doi. org/10.21037/tlcr-20-1130

Conflicts of Interest: All authors have completed the ICMJE uniform disclosure form (available at http://dx.doi. org/10.21037/tlcr-20-1130). The authors have no conflicts of interest to declare.

Ethical Statement: The authors are accountable for all aspects of the work in ensuring that questions related to the accuracy or integrity of any part of the work are appropriately investigated and resolved. All procedures performed in studies involving human participants were in accordance with the Helsinki Declaration (as revised in 2013). The study was approved by the ethics committee of Zhejiang Cancer Hospital. Informed consent was obtained from each patient.

Open Access Statement: This is an Open Access article distributed in accordance with the Creative Commons
Attribution-NonCommercial-NoDerivs 4.0 International License (CC BY-NC-ND 4.0), which permits the noncommercial replication and distribution of the article with the strict proviso that no changes or edits are made and the original work is properly cited (including links to both the formal publication through the relevant DOI and the license). See: https://creativecommons.org/licenses/by-nc-nd/4.0/.

\section{References}

1. Kim Y, Hammerman PS, Kim J, et al. Integrative and Comparative Genomic Analysis of Lung Squamous Cell Carcinomas in East Asian Patients. J Clin Oncol 2014;32:121-8.

2. Langer CJ, Obasaju C, Bunn P, et al. Incremental Innovation and Progress in Advanced Squamous Cell Lung Cancer: Current Status and Future Impact of Treatment. J Thorac Oncol 2016;11:2066-81.

3. Jiang $\mathrm{T}, \mathrm{Zhou} \mathrm{C}, \mathrm{Hu}$ J, et al. Combination immune checkpoint inhibitors with platinum-based chemotherapy in advanced non-small cell lung cancer: what's known and what's next. Transl Lung Cancer Res 2019;8:S447-50.

4. Reck M, Rodríguez Abreu D, Robinson AG, et al. Updated Analysis of KEYNOTE-024: Pembrolizumab Versus Platinum-Based Chemotherapy for Advanced NonSmall-Cell Lung Cancer With PD-L1 Tumor Proportion Score of 50\% or Greater. J Clin Oncol 2019;37:537-46.

5. Piper AJ, Sehgal K, Costa DB, et al. Can PD-L1 tumor proportion score be used as the key to unlocking the KEYNOTE studies of pembrolizumab in advanced lung cancer? Transl Lung Cancer Res. 2019;8:715-22.

6. Huang Y, Walsh RJ, Soo RA. The KEY to the end of the chemotherapy in advanced non-small cell lung cancer, or not yet? Transl Lung Cancer Res 2019;8:731-7.

7. Brahmer J, Reckamp KL, Baas P, et al. Nivolumab versus Docetaxel in Advanced Squamous-Cell Non-Small-Cell Lung Cancer. New Engl J Med 2015;373:123-35.

8. Rittmeyer A, Barlesi F, Waterkamp D, et al. Atezolizumab versus docetaxel in patients with previously treated non-small-cell lung cancer (OAK): a phase 3, openlabel, multicentre randomised controlled trial. Lancet 2017;389:255-65.

9. Herbst RS, Baas P, Kim D, et al. Pembrolizumab versus docetaxel for previously treated, PD-L1-positive, advanced non-small-cell lung cancer (KEYNOTE-010): a randomised controlled trial. Lancet 2016;387:1540-50.

10. Mok TSK, Wu Y, Kudaba I, et al. Pembrolizumab versus chemotherapy for previously untreated, PD-L1-expressing, 
locally advanced or metastatic non-small-cell lung cancer (KEYNOTE-042): a randomised, open-label, controlled, phase 3 trial. Lancet 2019;393:1819-30.

11. Hellmann MD, Ciuleanu T, Pluzanski A, et al. Nivolumab plus Ipilimumab in Lung Cancer with a High Tumor Mutational Burden. New Engl J Med 2018;378:2093-104.

12. Cristescu R, Mogg R, Ayers M, et al. Pan-tumor genomic biomarkers for PD-1 checkpoint blockade-based immunotherapy. Science 2018;362:r3593.

13. Kichenadasse G, Miners JO, Mangoni AA, et al. Association Between Body Mass Index and Overall Survival With Immune Checkpoint Inhibitor Therapy for Advanced Non-Small Cell Lung Cancer. JAMA Oncol 2020;6:512.

14. Matthew D. Hellmann, Tavi Nathanson, Hira Rizvi, et al. Genomic Features of Response to Combination Immunotherapy in Patients with Advanced Non SmallCell Lung Cancer. Cancer Cell 2018;33:843-152.e4.

15. Yates LR, Campbell PJ. Evolution of the cancer genome. Nat Rev Genet 2012;13:795-806.

16. Fang W, Ma Y, Yin JC, et al. Comprehensive Genomic Profiling Identifies Novel Genetic Predictors of Response to Anti-PD-(L)1 Therapies in Non-Small Cell Lung Cancer. Clin Cancer Res 2019;25:5015-26.

17. Paz-Ares L, Luft A, Vicente D, et al. Pembrolizumab plus Chemotherapy for Squamous Non-Small-Cell Lung Cancer. N Engl J Med 2018;379:2040-51.

18. Juergens RA, Mariano C, Jolivet J, et al. Real-world benefit of nivolumab in a Canadian non-small-cell lung cancer cohort. Curr Oncol 2018;25:384-92.

19. Felip E, Ardizzoni A, Ciuleanu T, et al. CheckMate 171: A phase 2 trial of nivolumab in patients with previously treated advanced squamous non-small cell lung cancer, including ECOG PS 2 and elderly populations. Eur J Cancer 2020;127:160-72.

20. Grossi F, Crinò L, Logroscino A, et al. Use of nivolumab in elderly patients with advanced squamous non-small-cell lung cancer: results from the Italian cohort of an expanded access programme. Eur J Cancer 2018;100:126-34.

21. Spigel DR, McCleod M, Jotte RM, et al. Safety, Efficacy, and Patient-Reported Health-Related Quality of Life and Symptom Burden with Nivolumab in Patients with Advanced Non-Small Cell Lung Cancer, Including Patients Aged 70 Years or Older or with Poor Performance Status (CheckMate 153). J Thorac Oncol 2019;14:1628-39.

22. Middleton G, Brock K, Savage J, et al. Pembrolizumab in patients with non-small-cell lung cancer of performance status 2 (PePS2): a single arm, phase 2 trial. Lancet Respir Med 2020;8:895-904.

23. Kytola V, Topaloglu U, Miller LD, et al. Mutational Landscapes of Smoking-Related Cancers in Caucasians and African Americans: Precision Oncology Perspectives at Wake Forest Baptist Comprehensive Cancer Center. Theranostics 2017;7:2914-23.

24. Gainor JF, Rizvi H, Jimenez Aguilar E, et al. Clinical activity of programmed cell death 1 (PD-1) blockade in never, light, and heavy smokers with non-small-cell lung cancer and PD-L1 expression $\geq 50 \%$. Ann Oncol 2020;31:404-11.

25. Rosell R, Carcereny E, Gervais R, et al. Erlotinib versus standard chemotherapy as first-line treatment for European patients with advanced EGFR mutation-positive non-small-cell lung cancer (EURTAC): a multicentre, open-label, randomised phase 3 trial. Lancet Oncol 2012;13:239-46.

26. Schwaederle M, Elkin SK, Tomson BN, et al. Squamousness: Next-generation sequencing reveals shared molecular features across squamous tumor types. Cell Cycle 2015;14:2355-61.

27. Cancer Genome Atlas Research Network. Comprehensive genomic characterization of squamous cell lung cancers. Nature 2012;489:519-25.

28. Rizvi NA, Hellmann MD, Snyder A, et al. Mutational landscape determines sensitivity to PD-1 blockade in nonsmall cell lung cancer. Science 2015;348:124-8.

29. Borghaei H, Paz-Ares L, Horn L, et al. Nivolumab versus Docetaxel in Advanced Nonsquamous Non-Small-Cell Lung Cancer. New Engl J Med 2015;373:1627-39.

30. Skoulidis F, Byers LA, Diao L, et al. Co-occurring Genomic Alterations Define Major Subsets of KRASMutant Lung Adenocarcinoma with Distinct Biology, Immune Profiles, and Therapeutic Vulnerabilities. Cancer Discov 2015;5:860-77.

31. Dong ZY, Zhong WZ, Zhang XC, et al. Potential Predictive Value of TP53 and KRAS Mutation Status for Response to PD-1 Blockade Immunotherapy in Lung Adenocarcinoma. Clin Cancer Res 2017;23:3012-24.

32. Kato S, Goodman A, Walavalkar V, et al. Hyperprogressors after Immunotherapy: Analysis of Genomic Alterations Associated with Accelerated Growth Rate. Clin Cancer Res 2017;23:4242-50.

33. Fuentes-Antrás J, Provencio M, Díaz-Rubio E. Hyperprogression as a distinct outcome after immunotherapy. Cancer Treat Rev 2018;70:16-21.

34. Zhang XC, Wang J, Shao GG, et al. Comprehensive 
genomic and immunological characterization of Chinese non-small cell lung cancer patients. Nat Commun 2019;10:1772.

35. Okamoto T, Takada K, Sato S, et al. Clinical and Genetic Implications of Mutation Burden in Squamous Cell Carcinoma of the Lung. Ann Surg Oncol 2018;25:1564-71.

36. Alexandrov LB, Nik-Zainal S, Wedge DC, et al. Deciphering Signatures of Mutational Processes Operative in Human Cancer. Cell Rep 2013;3:246-59.

37. Vogelstein B, Papadopoulos N, Velculescu VE, et al. Cancer Genome Landscapes. Science 2013;339:1546-58.

38. Rizvi H, Sanchez-Vega F, La K, et al. Molecular Determinants of Response to Anti-Programmed Cell Death (PD)-1 and Anti-Programmed Death-Ligand 1 (PD-L1) Blockade in Patients With Non-Small-Cell
Lung Cancer Profiled With Targeted Next-Generation Sequencing. J Clin Oncol 2018;36:633-41.

39. Rizvi NA, Hellmann MD, Snyder A, et al. Cancer immunology. Mutational landscape determines sensitivity to PD-1 blockade in non-small cell lung cancer. Science 2015;348:124-8.

40. Carbone DP, Reck M, Paz-Ares L, et al. First-Line Nivolumab in Stage IV or Recurrent Non-Small-Cell Lung Cancer. New Engl J Med 2017;376:2415-26.

41. Hellmann MD, Callahan MK, Awad MM, et al. Tumor Mutational Burden and Efficacy of Nivolumab Monotherapy and in Combination with Ipilimumab in Small-Cell Lung Cancer. Cancer Cell 2018;33:853-861.e4.

(English Language Editor: A. Kassem)
Cite this article as: $\mathrm{Xu} \mathrm{Y,} \mathrm{Li} \mathrm{H,} \mathrm{Huang} \mathrm{Z,} \mathrm{Chen} \mathrm{K,} \mathrm{Yu} \mathrm{X,}$ Sheng J, Zhang HH, Fan Y. Predictive values of genomic variation, tumor mutational burden, and PD-L1 expression in advanced lung squamous cell carcinoma treated with immunotherapy. Transl Lung Cancer Res 2020;9(6):2367-2379. doi: 10.21037/tlcr-20-1130 


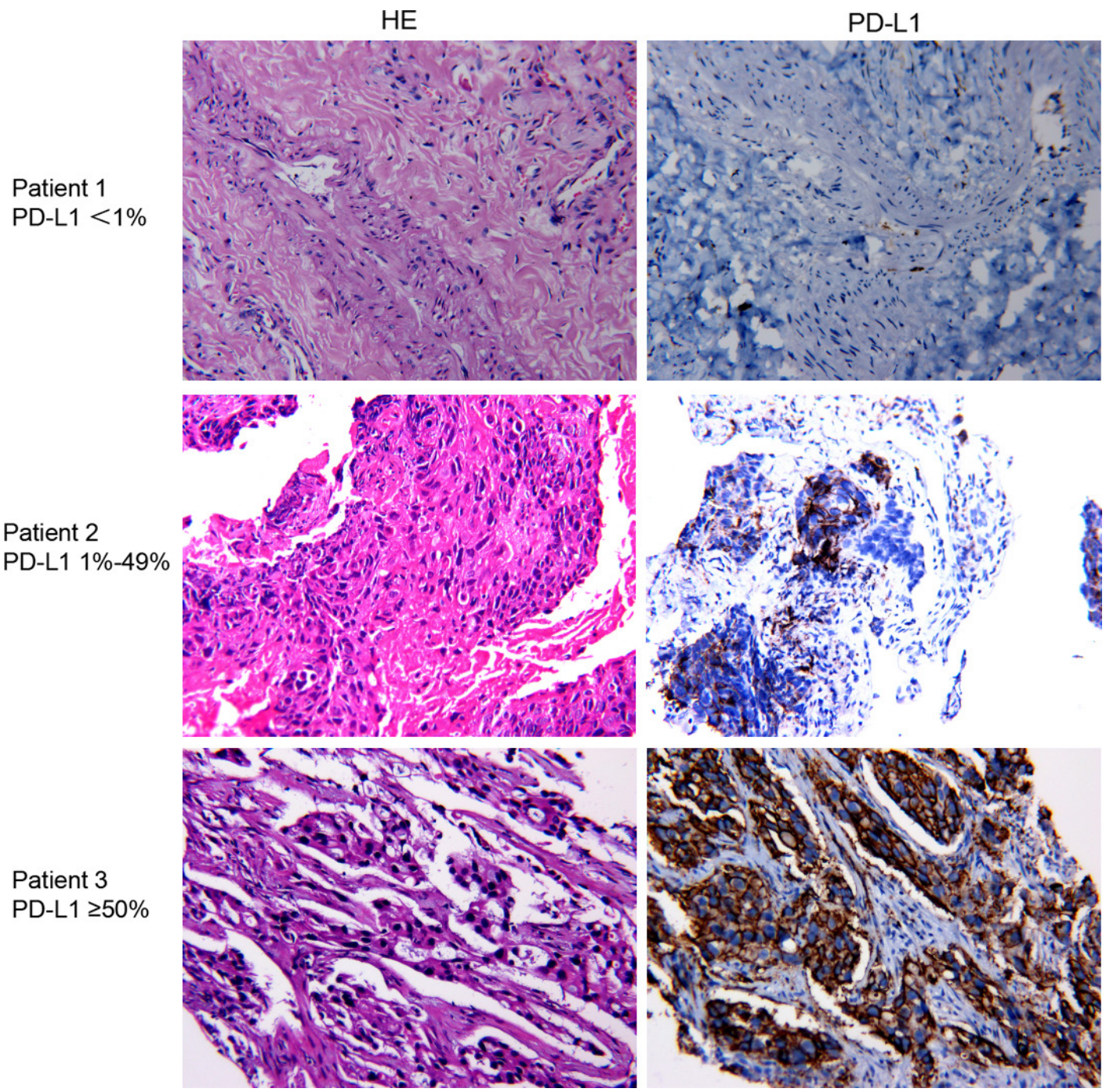

Figure S1 Representative IHC images. PD-L1 expression was tested by anti-human PD-L1 (Dako 22C3). The cutoff value was $<1 \%$ for PD-L1 positivity or negativity. The cutoff value was $\geq 50 \%$ for high expression level. IHC, immunohistochemical; PD-L1, programmed cell death ligand-1. 

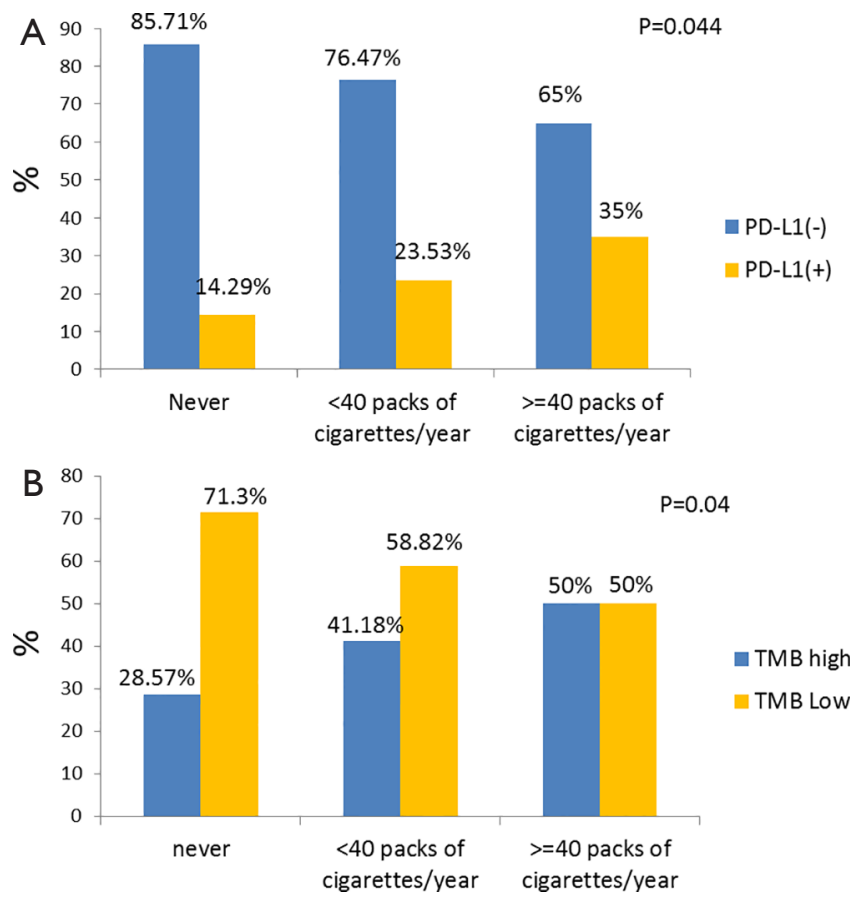

Figure S2 The relationship between smoking status, PD-L1 positivity, and TMB. (A) Patients with a history of heavy smoking ( $\geq 40$ pack-years) were more likely to have a higher $\mathrm{PD}-\mathrm{L} 1$ positive rate $(\mathrm{P}=0.044)$. The proportion of LUSC patients with positive PD-L1 increases with the addition of smoking pack-years. Positive PD-L1 expression was identified in $14.29 \%$ of never smokers, $23.53 \%$ of $<40$ pack-year smokers, and $35 \%$ of $\geq 40$ pack-year smokers. (B) Patients with a history of heavy smoking ( $\geq 40$ packyears) were more likely to have a higher TMB $(\mathrm{P}=0.04)$. The proportion of LUSC patients with high TMB increases with the addition of smoking pack-years. High TMB were identified in $28.57 \%$ of never smokers, $41.18 \%$ of $<40$ pack-year smokers, and $50 \%$ of $\geq 40$ pack-year smokers. PD-L1, programmed cell death ligand-1; TMB, tumor mutational burden; LUSC, lung squamous cell carcinoma. 
Table S1 Gene variations of included patients and their correlations with TMB and PD-L1 expression (N=64).

\begin{tabular}{|c|c|c|c|c|c|c|c|c|c|c|c|}
\hline \multirow[t]{2}{*}{ Gene } & \multirow[t]{2}{*}{ Total, N (\%) } & \multicolumn{2}{|c|}{ PD-L1 (-) } & \multicolumn{2}{|c|}{ PD-L1 (+) } & \multirow[t]{2}{*}{$P$ value } & \multicolumn{2}{|c|}{ TMB low* } & \multicolumn{2}{|c|}{ TMB low** } & \multirow[t]{2}{*}{$P$ value } \\
\hline & & $n$ & $\%$ & $\mathrm{n}$ & $\%$ & & $n$ & $\%$ & & N\% & \\
\hline \multicolumn{12}{|l|}{ TP53 mutation } \\
\hline Yes & 44 (68.8\%) & 30 & 68.18 & 14 & 31.82 & 0.5800 & 22 & 50.00 & 22 & 50.00 & 0.2638 \\
\hline No & 20 (31.2\%) & 15 & 75.00 & 5 & 25.00 & & 13 & 65.00 & 7 & 35.00 & \\
\hline \multicolumn{12}{|l|}{ CDKN2A mutation } \\
\hline Yes & $9(14.1 \%)$ & 6 & 66.67 & 3 & 33.33 & 0.2866 & 3 & 33.33 & 6 & 66.67 & 0.1129 \\
\hline No & 55 (85.9\%) & 39 & 70.91 & 16 & 29.09 & & 32 & 58.18 & 23 & 41.82 & \\
\hline \multicolumn{12}{|l|}{ KEAP1 mutation } \\
\hline Yes & $8(12.5 \%)$ & 4 & 50.00 & 4 & 50.00 & 0.1788 & 2 & 25.00 & 6 & 75.00 & 0.0639 \\
\hline No & 56 (87.5\%) & 41 & 73.21 & 15 & 26.79 & & 33 & 58.93 & 23 & 41.07 & \\
\hline \multicolumn{12}{|l|}{ CREBBP mutation } \\
\hline Yes & $7(10.9 \%)$ & 3 & 42.86 & 4 & 57.14 & 0.0885 & 3 & 42.86 & 4 & 57.14 & 0.2502 \\
\hline No & 57 (89.1\%) & 42 & 73.68 & 15 & 26.32 & & 32 & 56.14 & 25 & 43.86 & \\
\hline \multicolumn{12}{|l|}{ KRAS mutation } \\
\hline Yes & 7 (10.9\%) & 4 & 57.14 & 3 & 42.86 & 0.2324 & 5 & 71.43 & 2 & 28.57 & 0.2122 \\
\hline No & 57 (89.1\%) & 41 & 71.93 & 16 & 28.07 & & 30 & 52.63 & 27 & 47.37 & \\
\hline \multicolumn{12}{|l|}{ BIM mutation } \\
\hline Yes & $5(7.8 \%)$ & 4 & 80.00 & 1 & 20.00 & 0.3713 & 3 & 60.00 & 2 & 40.00 & 0.3485 \\
\hline No & 59 (92.2\%) & 41 & 69.49 & 18 & 30.51 & & 32 & 54.24 & 27 & 45.76 & \\
\hline \multicolumn{12}{|l|}{ AMER1 mutation } \\
\hline Yes & $4(6.2 \%)$ & 3 & 75.00 & 1 & 25.00 & 0.4243 & 1 & 25.00 & 3 & 75.00 & 0.2013 \\
\hline No & $60(93.8 \%)$ & 42 & 70.00 & 18 & 30.00 & & 34 & 56.67 & 26 & 43.33 & \\
\hline \multicolumn{12}{|l|}{ APC mutation } \\
\hline Yes & $4(6.2 \%)$ & 3 & 75.00 & 1 & 25.00 & 0.4243 & 2 & 50.00 & 2 & 50.00 & 0.3802 \\
\hline No & 60 (93.8\%) & 42 & 70.00 & 18 & 30.00 & & 33 & 55.00 & 27 & 45.00 & \\
\hline \multicolumn{12}{|l|}{ AR amplification } \\
\hline Yes & 17 (26.6\%) & 13 & 76.47 & 4 & 23.53 & 0.5167 & 9 & 52.94 & 8 & 47.06 & 0.8660 \\
\hline No & 47 (73.4\%) & 32 & 68.09 & 15 & 31.91 & & 26 & 55.32 & 21 & 44.68 & \\
\hline \multicolumn{12}{|l|}{ SOX2 amplification } \\
\hline Yes & 14 (21.9\%) & 9 & 64.29 & 5 & 35.71 & 0.5766 & 6 & 42.86 & 8 & 57.14 & 0.3144 \\
\hline No & $50(78.1 \%)$ & 36 & 72.00 & 14 & 28.00 & & 29 & 58.00 & 21 & 42.00 & \\
\hline PIK3CA amplification & & & & & & & & & & & \\
\hline Yes & $11(17.2 \%)$ & 6 & 54.55 & 5 & 45.45 & 0.2085 & 8 & 72.73 & 3 & 27.27 & 0.1157 \\
\hline No & $53(82.8 \%)$ & 39 & 73.58 & 14 & 26.42 & & 27 & 50.94 & 26 & 49.06 & \\
\hline EGFR amplification & & & & & & & & & & & \\
\hline Yes & $8(12.5 \%)$ & 5 & 62.50 & 3 & 37.50 & 0.2675 & 2 & 25.00 & 6 & 75.00 & 0.0639 \\
\hline No & $56(87.5 \%)$ & 40 & 71.43 & 16 & 28.57 & & 33 & 58.93 & 23 & 41.07 & \\
\hline RICTOR amplification & & & & & & & & & & & \\
\hline Yes & 7 (10.9\%) & 5 & 71.43 & 2 & 28.57 & 0.3363 & 5 & 71.43 & 2 & 28.57 & 0.2122 \\
\hline No & 57 (89.1\%) & 40 & 70.18 & 17 & 29.82 & & 30 & 52.63 & 27 & 47.37 & \\
\hline FGFR1 amplification & & & & & & & & & & & \\
\hline Yes & $6(9.4 \%)$ & 4 & 66.67 & 2 & 33.33 & 0.3398 & 3 & 50.00 & 3 & 50.00 & 0.3190 \\
\hline No & $58(90.6 \%)$ & 41 & 70.69 & 17 & 29.31 & & 32 & 55.17 & 26 & 44.83 & \\
\hline ZNF703 amplification & $5(7.8 \%)$ & 4 & 80.00 & 1 & 20.00 & 0.3713 & 3 & 60.00 & 2 & 40.00 & 0.3485 \\
\hline Yes & & & & & & & & & & & \\
\hline No & $59(92.2 \%)$ & 41 & 69.49 & 18 & 30.51 & & 32 & 54.24 & 27 & 45.76 & \\
\hline PIK3CA mutation or ar & ation & & & & & & & & & & \\
\hline Yes & 23 (35.9\%) & 15 & 65.22 & 8 & 34.78 & 0.5040 & 14 & 60.87 & 9 & 39.13 & 0.4568 \\
\hline No & $41(64.1 \%)$ & 30 & 73.17 & 11 & 26.83 & & 21 & 51.22 & 20 & 48.78 & \\
\hline
\end{tabular}

${ }^{*}$ TMB low, TMB $\leq 10.62$ (Mean TMB for all 64 subjects is 10.62); **TMB high, TMB >10.62. TMB, tumor mutational burden; PD-L1, programmed death ligand 1. 
Table S2 Univariate and multivariate analyses of clinical parameters on overall response and disease control (Logistic regression) (N=64)

\begin{tabular}{|c|c|c|c|c|c|c|}
\hline Factor & \multicolumn{3}{|c|}{ Univariate analysis } & \multicolumn{3}{|c|}{ Multivariate analysis } \\
\hline \multicolumn{7}{|l|}{ Overall response rate } \\
\hline Sex (male/female) & 0.607 & $0.093-3.962$ & 0.6020 & & & \\
\hline Age $(\geq 65 /<65)$ & 0.760 & $0.243-2.376$ & 0.6373 & & & \\
\hline Smoker ( $\geq 40$ pack-years/no) & 1.667 & $0.287-9.664$ & 0.0512 & & & \\
\hline BMI (18.5-24/<18.5) & 0.722 & $0.140-3.731$ & 0.5085 & & & \\
\hline BMI $(\geq 24 /<18.5)$ & 0.235 & $0.034-1.631$ & 0.0883 & & & \\
\hline ECOG $(1-2 / 0)$ & 0.009 & $0.001-0.057$ & 0.0000 & 0.027 & $0.005-0.142$ & $<0.0001$ \\
\hline Liver metastases (yes/no) & 0.933 & $0.252-3.452$ & 0.9176 & & & \\
\hline Radiotherapy (yes/no) & 0.120 & $0.034-0.429$ & 0.0011 & 0.241 & $0.045-1.280$ & 0.0949 \\
\hline Lines of therapy (yes/no) & $<0.001$ & $<0.001->999.999$ & 0.9402 & & & \\
\hline PD-L1 (positive/negative) & 0.880 & $0.275-2.815$ & 0.8297 & & & \\
\hline TMB (>10.62/ $\leq 10.62)$ & 1.520 & $0.518-4.466$ & 0.4459 & & & \\
\hline \multicolumn{7}{|l|}{ Disease control rate } \\
\hline Sex (male/female) & $<0.001$ & $<0.001->999.999$ & 0.9661 & & & \\
\hline Age $(\geq 65 /<65)$ & 1.152 & $0.306-4.338$ & 0.8349 & & & \\
\hline Dyslipidemia (yes/no) & 1.261 & $0.359-4.432$ & 0.7178 & & & \\
\hline Brain metastases (yes/no) & 0.652 & $0.114-3.715$ & 0.6299 & & & \\
\hline Liver metastases (yes/no) & 0.293 & $0.076-1.135$ & 0.0756 & & & \\
\hline Radiotherapy (yes/no) & 1.261 & $0.359-4.432$ & 0.7178 & & & \\
\hline Lines of therapy (yes/no) & 0.184 & $0.048-0.709$ & 0.0139 & 0.425 & $0.050-3.640$ & 0.4345 \\
\hline PD-L1 (positive/negative) & 0.412 & $0.081-2.091$ & 0.2845 & & & \\
\hline 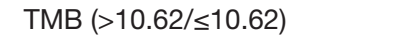 & 5.400 & $1.076-27.09$ & 0.0404 & 13.109 & $1.429-120.2$ & 0.0229 \\
\hline
\end{tabular}

BMI, body mass index; ECOG, Eastern Cooperative Oncology Group; PD-L1, programmed cell death ligand-1; TMB, tumor mutational burden. 
Table S3 Univariate and multivariate analyses of immunotherapy-only patients on Progression-Free Survival and Overall Survival (Cox regression) (N=53)

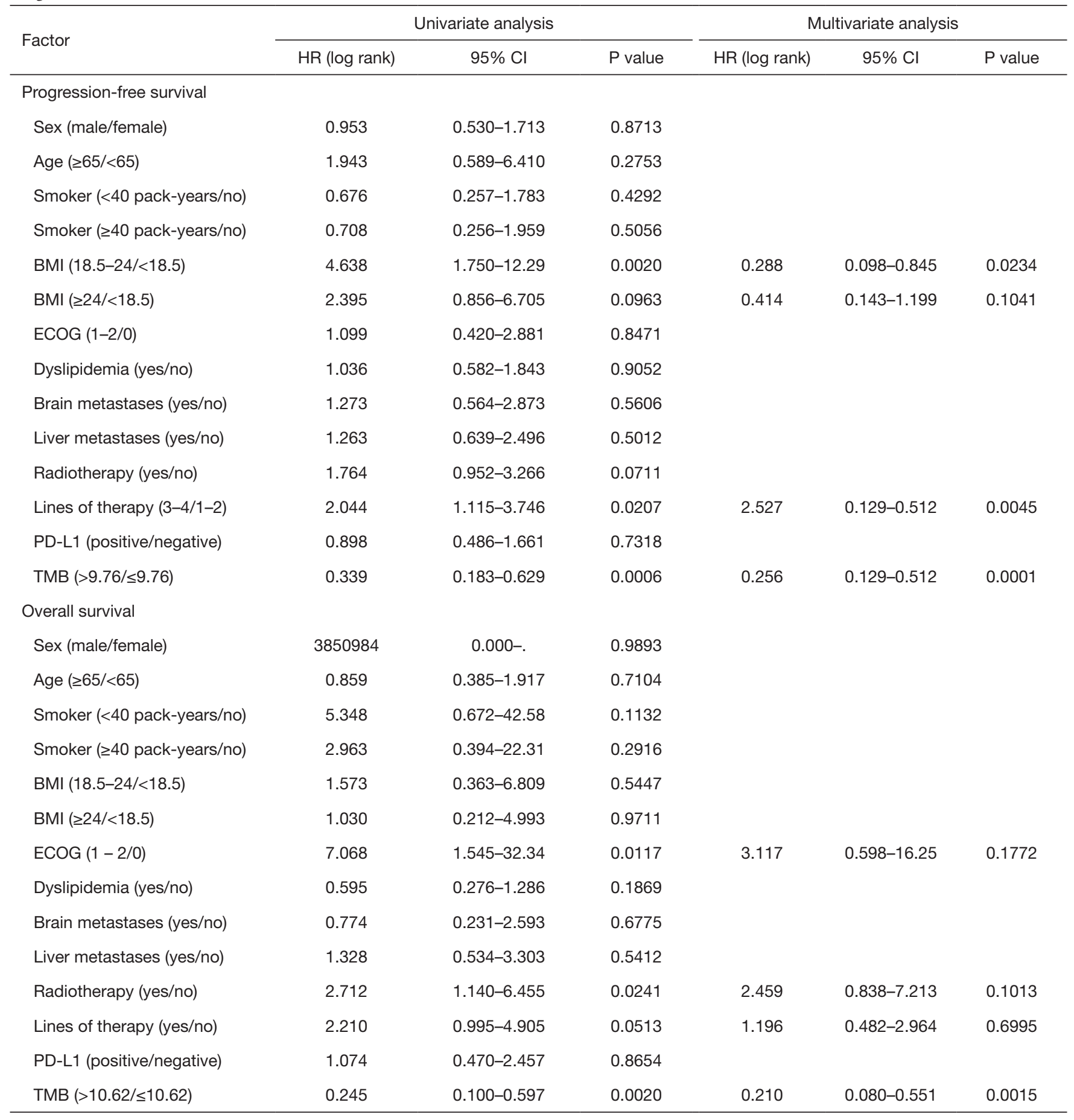

BMI, body mass index; ECOG, Eastern Cooperative Oncology Group; PD-L1, programmed cell death ligand-1; TMB, tumor mutational burden. 
Table S4 Univariate analyses of TMB and PD-L1 on overall survival and progression-free survival (Cox regression)

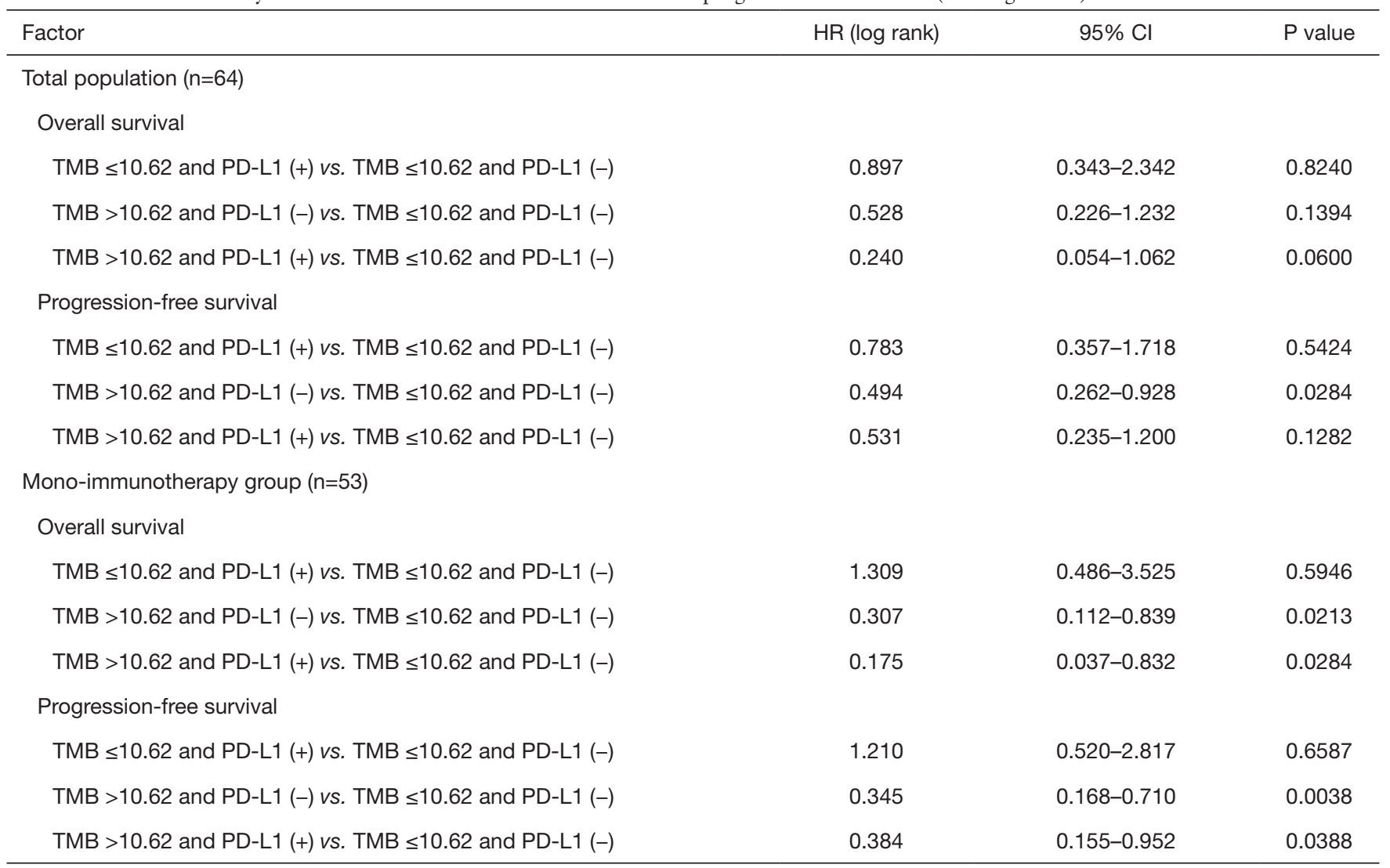

TMB, tumor mutational burden; PD-L1, programmed cell death ligand-1; 
Table S5 Univariate and multivariate analyses of gene variations on progression-free survival and overall survival (Cox regression) (N=64)

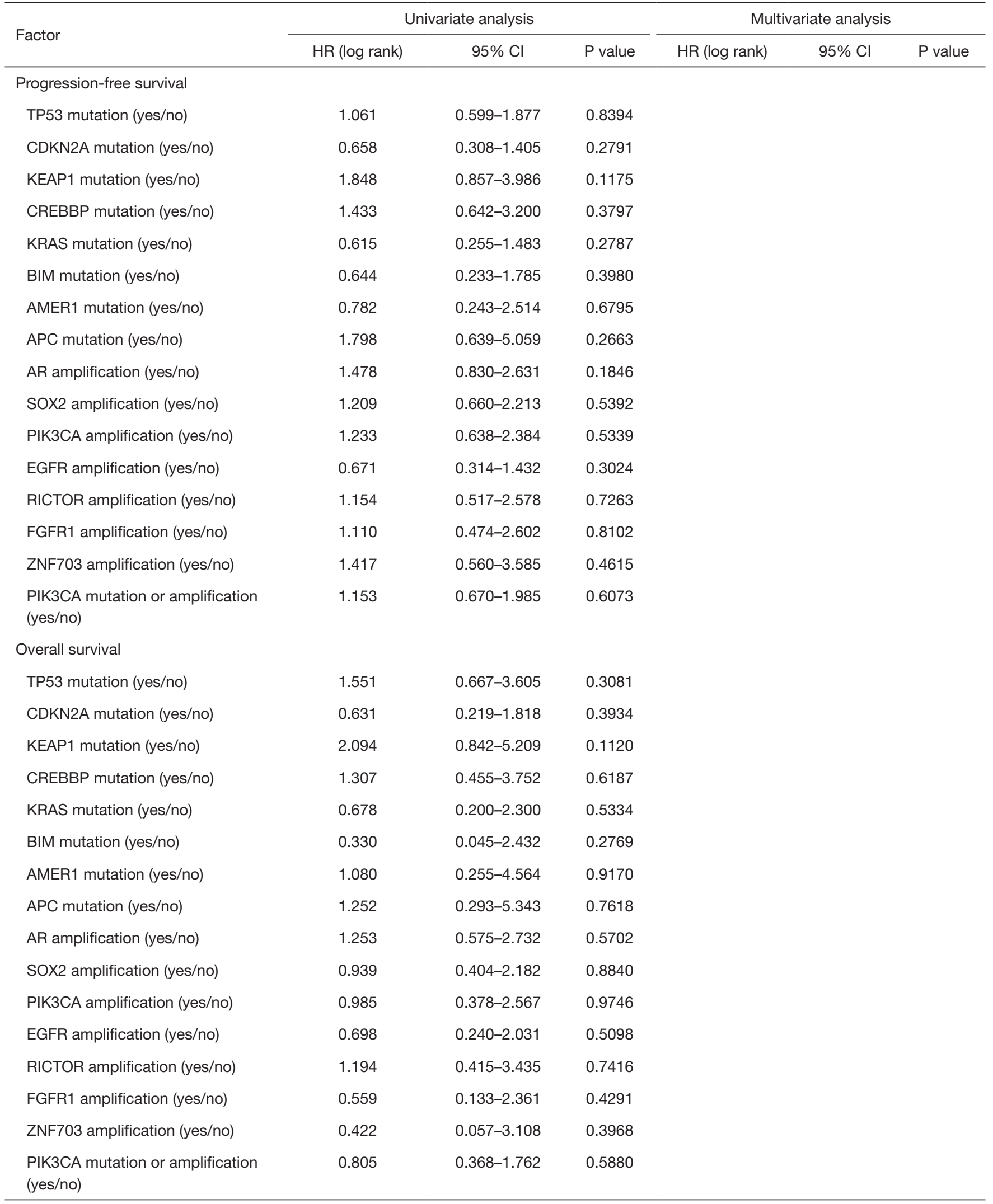


Table S6 Univariate and multivariate analyses of gene variations on overall response and disease control (Logistic regression) (N=64)

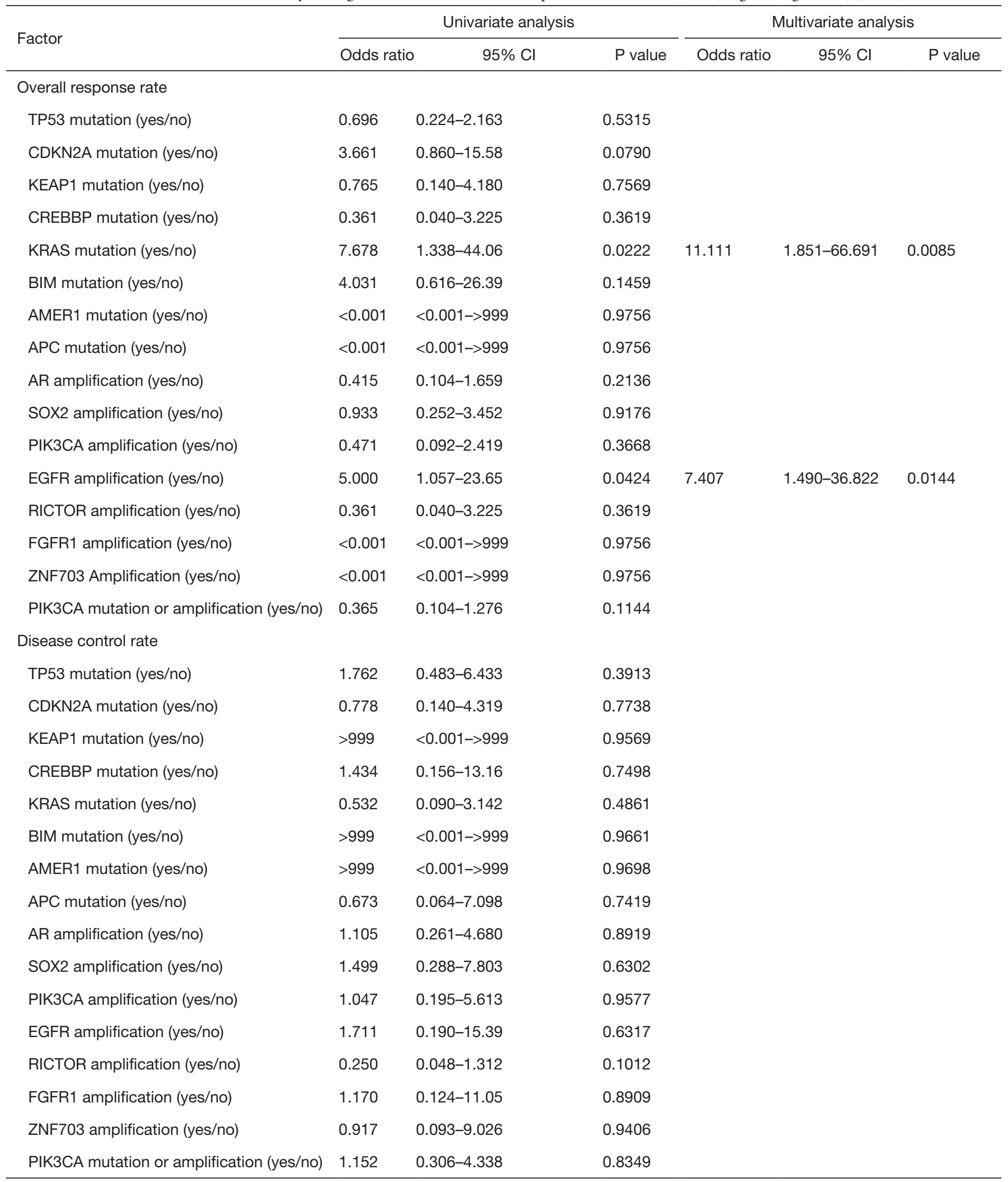

